\title{
LIMIT THEOREMS FOR COUNTING LARGE CONTINUED FRACTION DIGITS
}

\author{
MARC KESSEBÖHMER AND TANJA SCHINDLER
}

\begin{abstract}
We establish a central limit theorem for counting large continued fraction digits $\left(a_{n}\right)$, i.e. we count occurances $\left\{a_{n}>b_{n}\right\}$, where $\left(b_{n}\right)$ is a sequence of positive integers. Our result improves a similar result by Philipp which additionally assumes that $b_{n}$ tends to infinity. Moreover, we give a refinement of the famous Borel-Bernstein Theorem for continued fractions regarding the event that the $n$-th continued fraction digit lies infinitely often between $d_{n}$ and $d_{n}\left(1+1 / c_{n}\right)$ for given sequences $\left(c_{n}\right)$ and $\left(d_{n}\right)$. Also for these sets we obtain a central limit theorem. As an interesting side result we determine the first $\phi$-mixing coefficient for the Gauss system explicitly.
\end{abstract}

\section{INTRODUCTION AND STATEMENT OF RESULTS}

Throughout the paper, for any irrational number $\omega \in \mathbb{R} \backslash \mathbb{Q}$ we will denote its unique infinite regular continued fraction expansion by $\left[a_{0}(\omega) ; a_{1}(\omega), a_{2}(\omega), \ldots\right]$, where

$$
\omega=a_{0}(\omega)+\frac{1}{a_{1}(\omega)+\frac{1}{a_{2}(\omega)+\frac{1}{\ddots}}}=:\left[a_{0}(\omega) ; a_{1}(\omega), a_{2}(\omega), \ldots\right] .
$$

In case that $a_{0}(\omega)=0$ we write $\omega=\left[a_{1}(\omega), a_{2}(\omega), \ldots\right]$. We may also express this algorithm restricted to $I:=[0,1)$ by the Gauss map $G: I \rightarrow I$,

$$
G(\omega):= \begin{cases}1 / \omega-\lfloor 1 / \omega\rfloor, & \omega \neq 0, \\ 0, & \omega=0 .\end{cases}
$$

With $G^{0}:=\operatorname{id}$ and $G^{n}:=G \circ G^{n-1}, n \geq 1$ we obtain the sequence of digits $a_{n}(\omega):=\left\lfloor 1 / G^{n-1}(\omega)\right\rfloor$, $n \in \mathbb{N}$. The algorithm will terminate in $n \in \mathbb{N}$ only for rational numbers $\omega$, whenever $G^{n}(\omega)=0$ for the first time; in this way we obtain the finite continued fraction expansion of $\omega \in \mathbb{Q}$. In this case we write $\omega=\left[a_{1}(\omega), a_{2}(\omega), \ldots, a_{n}(\omega)\right]$.

The transformation $G$ does not preserve the Lebesgue measure restricted to $I$ denoted by $\lambda_{I}$ (cf. DK02, Chapter 1.3.3]). However, Gauss found a $G$-invariant measure $\mathfrak{m}$ which is equivalent to $\lambda$ with density $h(x)=1 /((x+1) \log 2), x \in I$ (cf. IK09, Chapter 1.2.2]). The dynamical system $\left(I,\left.\mathcal{B}\right|_{I}, G, \mathfrak{m}\right)$ is in fact ergodic and even a lot stronger mixing conditions hold, see for example [Bra05] and Section 2

Based on these mixing properties Philipp proved a central limit theorem (CLT) counting large entries of the continued fraction expansion, see [Phi70, Theorem 2]. He also gave a remainder term which was later improved by Zuparov giving, Zup81. Philipp and Webb PW73, improved the result by proving a functional limit theorem in the function space $\mathbb{D}[0,1]$.

To state this theorem by Philipp we let $\mathbb{V}(X):=\mathbb{E}\left((X-\mathbb{E}(X))^{2}\right)$ denote the variance of the random variable $X$.

Theorem 1.1 ( $\left[\right.$ Phi70, Theorem 2]). Consider a sequence of positive reals $\left(b_{n}\right)_{n \in \mathbb{N}}$. If

$$
\lim _{n \rightarrow \infty} b_{n}=\infty \quad \text { and } \quad \sum_{n \in \mathbb{N}} \frac{1}{b_{n}}=\infty,
$$

Date: December 31, 2018

2010 Mathematics Subject Classification. Primary: 11K50 Secondary: 60F20, 60F05 .

Key words and phrases. continued fractions, zero-one laws, central limit theorem, $\phi$-mixing, Diophantine approximation. This research was supported by the German Research Foundation (DFG) grant Renewal Theory and Statistics of Rare Events in Infinite Ergodic Theory (Geschäftszeichen KE 1440/2-1). TS was supported by the Studienstiftung des Deutschen Volkes. 
then for $S_{n}:=\sum_{k=1}^{n} \mathbb{1}_{\left\{a_{k}>b_{k}\right\}}$ we have

$$
\lim _{n \rightarrow \infty} \mathfrak{m}\left(\frac{S_{n}-\mathbb{E}\left(S_{n}\right)}{\sqrt{\mathbb{V}\left(S_{n}\right)}}<z\right)=\frac{1}{\sqrt{2 \pi}} \int_{-\infty}^{z} e^{-t^{2} / 2} \mathrm{~d} t .
$$

The necessary conditions in this theorem are intimately connected with the following classical zero-one law by Borel and Bernstein Bor09, Ber11, Ber12.

Theorem 1.2 (Borel-Bernstein Theorem). Consider a sequence of positive reals $\left(b_{n}\right)_{n \in \mathbb{N}}$. Then $a_{n}(\omega) \geq$ $b_{n}$ holds infinitely often with Lebesgue measure 0 or 1 , according as the series $\sum_{n \in \mathbb{N}} 1 / b_{n}$ converges or diverges.

If one compares the two theorems stated above, the additional condition $\lim _{n \rightarrow \infty} b_{n}=\infty$ in the CLT seems to be artificial. We might also compare the assumptions of Theorem [1.1 with the necessary conditions for the CLT to hold in the case that $\left(X_{i}\right):=\left(\mathbb{1}_{A_{i}}\right)$ is a sequence of independent random variables (like for the Lüroth system, cf. for example Gal72]) with the same distribution function. We can then make use of Lindeberg's condition. That is we assume that for all $\epsilon>0$ we have

$$
\lim _{n \rightarrow \infty} \frac{1}{\mathbb{V}\left(S_{n}\right)} \cdot \sum_{i=1}^{n} \mathbb{E}\left(\left(X_{i}-\mathbb{E}\left(X_{i}\right)\right)^{2} \cdot \mathbb{1}_{\left\{\left|X_{i}-\mathbb{E}\left(X_{i}\right)\right|>\epsilon \cdot \sqrt{\mathbb{V}\left(S_{n}\right)}\right\}}\right)=0 .
$$

We find that this condition is in fact equivalent to $\lim _{n \rightarrow \infty} \mathbb{V}\left(S_{n}\right)=\infty$ which in the i.i.d. case is equivalent to $\lim _{n \rightarrow \infty} \sum_{i=1}^{n} \mathfrak{m}\left(A_{i}\right) \cdot \mathfrak{m}\left(A_{i}^{c}\right)=\infty$. This can be seen as follows: On the one hand, the condition $\lim _{n \rightarrow \infty} \mathbb{V}\left(S_{n}\right)=\infty$ combined with the fact that $X_{i}-\mathbb{E}\left(X_{i}\right) \in[-1,1]$ implies for all $\epsilon>0$ and all $n$ sufficiently large that we have $\left\{\left|X_{i}-\mathbb{E}\left(X_{i}\right)\right|>\epsilon \cdot \sqrt{\mathbb{V}\left(S_{n}\right)}\right\}=\varnothing$; hence (1) holds. On the other hand, if $\lim _{n \rightarrow \infty} \mathbb{V}\left(S_{n}\right)<\infty$ and $\sum_{n=1}^{\infty} \mathfrak{m}\left(A_{n}\right)=\infty$, then there exists $\epsilon>0$ sufficiently small such that

$$
\mathbb{E}\left(\left(X_{i}-\mathbb{E}\left(X_{i}\right)\right)^{2} \cdot \mathbb{1}_{\left\{\left|X_{i}-\mathbb{E}\left(X_{i}\right)\right|>\epsilon \cdot \sqrt{\mathbb{V}\left(S_{n}\right)}\right\}}\right)>0
$$

for some $i \in \mathbb{N}$ and arbitrarily large $n \in \mathbb{N}$. Consequently, (1) fails to hold.

It is the purpose of this paper to prove the following general CLT for indicator functions measurable with respect to the continued fraction digits requiring only the assumptions necessary for the i.i.d. case.

Theorem 1.3. Let $\left(A_{n}\right)_{n \in \mathbb{N}}$ be a sequence of events such that $A_{n} \in \sigma\left(a_{n}\right)$ for all $n \in \mathbb{N}$. Suppose

$$
V_{n}:=\sum_{k=1}^{n} \mathfrak{m}\left(A_{k}\right) \cdot \mathfrak{m}\left(A_{k}^{c}\right) \rightarrow \infty
$$

Then for $S_{n}:=\sum_{k=1}^{n} \mathbb{1}_{A_{k}}$ we have

$$
\lim _{n \rightarrow \infty} \mathfrak{m}\left(\frac{S_{n}-\mathbb{E}\left(S_{n}\right)}{\sqrt{\mathbb{V}\left(S_{n}\right)}}<z\right)=\frac{1}{\sqrt{2 \pi}} \int_{-\infty}^{z} e^{-t^{2} / 2} \mathrm{~d} t
$$

We remark here that to provide error terms as in Phi70 and Zup81, or to prove that for $S_{n}$ a functional CLT as in PW73 holds follows along the same lines as in the original papers and will be omitted.

From this theorem one can easily obtain the following improvement of Philipp's Theorem 1.1

Theorem 1.4. Consider a sequence of positive reals $\left(b_{n}\right)_{n \in \mathbb{N}}$. If

$$
\sum_{n \in \mathbb{N}} \frac{1}{b_{n}}=\infty
$$

then for $S_{n}:=\sum_{k=1}^{n} \mathbb{1}_{\left\{a_{k}>b_{k}\right\}}$ we have

$$
\lim _{n \rightarrow \infty} \mathfrak{m}\left(\frac{S_{n}-\mathbb{E}\left(S_{n}\right)}{\sqrt{\mathbb{V}\left(S_{n}\right)}}<z\right)=\frac{1}{\sqrt{2 \pi}} \int_{-\infty}^{z} e^{-t^{2} / 2} \mathrm{~d} t .
$$

One of the main ingredients for the proof of Theorem 1.3 is to prove that $\lim _{n \rightarrow \infty} \mathbb{V}\left(S_{n}\right)=\infty$ is equivalent to $\lim _{n \rightarrow \infty} \sum_{i=1}^{n} \mathfrak{m}\left(A_{i}\right) \cdot \mathfrak{m}\left(A_{i}^{c}\right)=\infty$. We will do this by making use of the $\phi$ - and $\psi$-mixing properties of the continued fraction digits, in particular we will give the precise value of the first $\phi$ mixing coefficient (Lemma 2.3) which might be of independent interest. In Remark 4.2 we will make clear that this estimate of the first $\phi$-mixing coefficient is a real improvement as the known estimates for the $\phi$-mixing coefficients are indeed not sufficient to prove the above equivalence. 
1.1. Central limit theorems and zero-one laws for intervals. The above stated theorems are all dealing with the sets $\left\{a_{n}>b_{n}\right\}$. To broaden the picture we will consider in this section the more general setting of $a_{n}$ hitting a certain interval. Results in this spirit have been proven for independent random variables in the context of Lüroth expansions by Galambos in Gal72.

We start with giving a CLT which can also be deduced from Theorem 1.3.

Theorem 1.5. Let $\left(c_{n}\right)_{n \in \mathbb{N}}$ be an arbitrarily chosen sequence of positive real numbers and $\left(d_{n}\right)_{n \in \mathbb{N}}$ be a sequences of positive integers. Suppose that either

(A) $A_{n}:=\left\{a_{n}=d_{n}\right\}$ with $\sum_{n \in \mathbb{N}} 1 / d_{n}^{2}=\infty$,

(B) $A_{n}:=\left\{d_{n} \leq a_{n} \leq d_{n} \cdot\left(1+\frac{1}{c_{n}}\right)\right\}$ with $\sum_{n: d_{n}>1} 1 /\left(c_{n} d_{n}\right)=\infty$ or $\sum_{n: d_{n}>1} 1 / d_{n}^{2}=\infty$ or $\sum_{n: d_{n}=1} c_{n}=\infty$,

(C) $A_{n}:=\left\{d_{n}<a_{n} \leq d_{n} \cdot\left(1+\frac{1}{c_{n}}\right)\right\}$ with $\sum_{n: c_{n} \leq d_{n}} 1 /\left(c_{n} d_{n}\right)=\infty$.

Then for $S_{n}:=\sum_{k=1}^{n} \mathbb{1}_{A_{k}}$ the CLT in (3) holds.

In a similar way we can give zero-one laws as an analog to the Borel-Bernstein theorem.

Theorem 1.6. Let $\left(c_{n}\right)_{n \in \mathbb{N}}$ be a sequence of positive real numbers and $\left(d_{n}\right)_{n \in \mathbb{N}}$ be a sequences of positive integers. Suppose that either

(A) $A_{n}:=\left\{a_{n}=d_{n}\right\}$ and $\Gamma:=\sum_{n \in \mathbb{N}} 1 / d_{n}^{2}$,

(B) $A_{n}:=\left\{d_{n} \leq a_{n} \leq d_{n} \cdot\left(1+\frac{1}{c_{n}}\right)\right\}$ and $\Gamma:=\max \left\{\sum_{n \in \mathbb{N}} \frac{1}{c_{n} d_{n}}, \sum_{n \in \mathbb{N}} \frac{1}{d_{n}^{2}}\right\}$,

(C) $A_{n}:=\left\{d_{n}<a_{n} \leq d_{n} \cdot\left(1+\frac{1}{c_{n}}\right)\right\}$ and $\Gamma:=\sum_{n: c_{n} \leq d_{n}} 1 /\left(c_{n} d_{n}\right)$.

Then $A_{n}$ holds infinitely often with Lebesgue measure 0 or 1 , according as $\Gamma$ is finite or not.

Regarding (A) we remark that for $d_{n}:=\lfloor\sqrt{n \log (n)}\rfloor$ there are almost surely infinitely many values of $n$ such that $a_{n}=d_{n}$ and for $e_{n}:=\lfloor\sqrt{n} \log (n)\rfloor$ there are almost surely only finitely many values of $n$ such that $a_{n}=e_{n}$. Particularly, if $\left(d_{n}\right)$ is bounded, then almost surely $a_{n}=d_{n}$ infinitely often.

Using a dynamical Borel-Cantelli lemma by Philipp, see [Phi67, Theorem 3], the results can be proven in a similar way as in the i.i.d. case, see Section 3 .

The condition for a Lebesgue measure 1 set in Theorem 1.6 differs in case (B) from the condition for a CLT. Indeed, the condition for a zero-one law is $\sum_{n=1}^{\infty} \mathfrak{m}\left(A_{n}\right)=\infty$, see Section 3 and particularly Lemma 3.1 which will be applied to prove the zero-one laws. In contrast to this, the condition for the CLT is $\sum_{n=1}^{\infty} \mathfrak{m}\left(A_{n}\right) \cdot \mathfrak{m}\left(A_{n}^{c}\right)=\infty$ and in this case we also have to ensure that $\mathfrak{m}\left(A_{n}^{c}\right)$ remains large enough.

A refined study of the Lebesgue measure zero sets may also be of interest. In fact, in WW08 the Hausdorff dimension of $\left\{x: a_{n}(x) \geq b_{n}\right.$ infinitely often $\}$ was subject of study for those $\left(b_{n}\right)$ for which the above set has 0 Lebesgue measure. These results could be carried over to the limsup sets considered in this paper. We also note here that the Hausdorff dimension of similar sets, namely with certain restriction on the continued fraction digits has been widely studied, for example for the set $\left\{x: a_{n} \in\{1,2\}\right.$ for all $\left.n \in \mathbb{N}\right\}$ in [JP18, and previous works like KZ06, KS07. Also sets concerning restrictions like $\left\{x: s_{n}<a_{n}(x) \leq s_{n} t_{n}\right.$ for all $\left.n \in \mathbb{N}\right\}$ with $\left(s_{n}\right),\left(t_{n}\right)$ being sequences of reals with $\left(s_{n}\right)$ tending to infinity have been studied in [FLWW09] and [LR16] as a tool to determine the fast Khintchine spectrum.

1.2. Khintchine's Theorem and zero-one laws for associated random variables. Inspired by Theorem 1.6 we will next state analogous results to Khintchine's famous zero-one law for Diophantine approximation which can be stated as follows Khi35].

Theorem 1.7 (Khintchine's Theorem). Let $k: \mathbb{N} \rightarrow(0, \infty)$ be such that $(n \cdot k(n)), n \in \mathbb{N}$ is nonincreasing. Then we have that for infinitely many $q \in \mathbb{N}$ there exists $p \in \mathbb{N}$ with greatest common divisor $(p, q)=1$ such that

$$
\left|x-\frac{p}{q}\right| \leq \frac{k(q)}{q}
$$

holds with Lebesgue measure 0 or 1 , according as $\sum_{n \in \mathbb{N}} k(n)$ is finite or not. 
Next, we define random variables that bridge the continued fraction digits $\left(a_{n}\right)$ of an irrational number to its Diophantine properties (see e.g. [IK09, Chapter 1.2.1]).

Lemma 1.8. Fix $x:=\left[a_{0} ; a_{1}, \ldots\right] \in \mathbb{R} \backslash \mathbb{Q}$. Then with $p_{-1}:=1, p_{0}:=a_{0}, q_{-1}:=0, q_{0}:=1$,

$$
p_{n}:=a_{n} p_{n-1}+p_{n-2}, \quad q_{n}:=a_{n} q_{n-1}+q_{n-2} \text { and } r_{n}:=\frac{1}{G^{n-1}}=\left[a_{n} ; a_{n+1}, a_{n+2}, \ldots\right]
$$

we have $q_{k} p_{k-1}-p_{k} q_{k-1}=(-1)^{k}$,

$$
x=\frac{p_{n-1} r_{n}+p_{n-2}}{q_{n-1} r_{n}+q_{n-2}} \text { and } \frac{p_{n}}{q_{n}}=a_{0}+\frac{1}{a_{1}+\frac{1}{a_{2}+\frac{1}{\ddots \cdot+\frac{1}{a_{n}}}}}=\left[a_{0} ; a_{1}, \ldots, a_{n}\right] .
$$

Setting

$$
y_{n}:=\frac{q_{n}}{q_{n-1}},
$$

for $n \in \mathbb{N}$, we have $q_{n}=y_{1} \cdots y_{n}$ and $y_{n}=\left[a_{n} ; a_{n-1}, \ldots, a_{1}\right]=a_{n}+y_{n-1}, n \in \mathbb{N}$. Further define

$$
u_{n}:=q_{n-1}^{-2}\left|x-\frac{p_{n-1}}{q_{n-1}}\right|^{-1} \text {. }
$$

The random variable $u_{n}$ is crucial in the context of Diophantine approximations. Recall the well-known estimate

$$
\frac{1}{q_{n-1}\left(q_{n}+q_{n-1}\right)}<\left|x-\frac{p_{n-1}}{q_{n-1}}\right|<\frac{1}{q_{n-1} q_{n}} .
$$

For a comprehensive account we refer to [DK02, Chapter 5], BvdPSZ14, [KMS16] or [K09]. Even more, Khintchine's Theorem 1.7 can be reformulated in terms of continued fraction entries as follows.

Lemma 1.9. Let $k: \mathbb{N} \rightarrow(0, \infty)$ be such that $n \cdot k(n)$ is non-increasing. Then

$$
\left|x-\frac{p_{i}}{q_{i}}\right|=\frac{1}{u_{i} \cdot q_{i}^{2}} \leq \frac{k\left(q_{i}\right)}{q_{i}}
$$

holds for infinitely many $i \in \mathbb{N}$ with Lebesgue measure 0 or 1 , according as $\sum_{n \in \mathbb{N}} k(n)$ is finite or not.

This can be easily deduced following the proof of Khintchine's Theorem in [Khi64, Theorem 32].

As we will see in the next lemma, the difference between the above defined variables and $a_{n}$ is bounded, which enables us to prove a theorem related to the continued fractions entries.

Lemma 1.10. Let the random variables $\left(r_{n}\right)_{n \in \mathbb{N}},\left(y_{n}\right)_{n \in \mathbb{N}}$, and $\left(u_{n}\right)_{n \in \mathbb{N}}$ be defined as above. Then

(A) $a_{n} \leq r_{n}<a_{n}+1$,

(B) $a_{n} \leq y_{n}<a_{n}+1$,

(C) $a_{n}<u_{n}<a_{n}+2$.

Proof. The inequalities (A) and (B) are immediate, (C) follows from Eq. (7).

For the associated random variables $\left(r_{n}\right),\left(y_{n}\right)$, and $\left(u_{n}\right)$ a Borel-Bernstein Theorem also holds. This follows immediately from an application of Lemma 1.10 and [K09, Corollary 1.3.17] to the Borel-Bernstein Theorem.

Our next theorem will give an analogous statement to Theorem 1.6 for the associated random variables $\left(r_{n}\right),\left(y_{n}\right)$, and $\left(u_{n}\right)$.

Theorem 1.11 (Corollary to Theorem 1.6). Let $\left(r_{n}\right)_{n \in \mathbb{N}},\left(y_{n}\right)_{n \in \mathbb{N}}$, and $\left(u_{n}\right)_{n \in \mathbb{N}}$ be the random variables associated to the continued fraction digits, as defined in Lemma 1.8 and Eq. (5) and (6). Further, let $\left(c_{n}\right)_{n \in \mathbb{N}}$ and $\left(d_{n}\right)_{n \in \mathbb{N}}$ be sequences of positive real numbers such that there exists $\epsilon>0$ fulfilling $c_{n} \leq d_{n} /(3+\epsilon)$ for all $n \in \mathbb{N}$ in case that we consider $r_{n}$ or $y_{n}$ and $c_{n} \leq d_{n} /(4+\epsilon)$ for all $n \in \mathbb{N}$ in case that we consider $u_{n}$. Then each of the corresponding inequalities

$$
d_{n} \leq r_{n} \leq d_{n}\left(1+1 / c_{n}\right), \quad d_{n} \leq y_{n} \leq d_{n}\left(1+1 / c_{n}\right), \quad d_{n} \leq u_{n} \leq d_{n}\left(1+1 / c_{n}\right)
$$

holds infinitely often with Lebesgue measure 0 or 1 , according as $\sum_{n \in \mathbb{N}} 1 /\left(c_{n} d_{n}\right)$ is finite or not. 
Remark 1.12 Other than in Theorem 1.6 we have here the additional condition that $c_{n} \leq d_{n} /(3+\epsilon)$ or $c_{n} \leq d_{n} /(4+\epsilon)$ respectively. Proving Theorem 1.11 as a corollary to Theorem 1.6 with the help of Lemma 1.10 makes this restriction necessary. Following this proof one might see that the condition can be relaxed to $c_{n} \leq d_{n} /(1+\epsilon)$ or $c_{n} \leq d_{n} /(2+\epsilon)$ respectively if we require $d_{n} \in \mathbb{N}$. However, it would be interesting if one could state the above theorem also for comparatively small intervals $\left[d_{n}, d_{n}\left(1+1 / c_{n}\right)\right]$, i.e. intervals with large $c_{n}$.

\section{Mixing PROPERTIES}

Our results will depend crucially on the mixing properties of the continued fraction digits. To explain this we first introduce the classical notion of $\phi$ - and $\psi$-mixing.

Definition 2.1 Let $(\Omega, \mathcal{A}, \mathbb{P})$ be a probability space and $\mathcal{C}, \mathcal{D} \subset \mathcal{A}$ two $\sigma$-fields, then the following quantities measure the dependence of the sub- $\sigma$-fields.

$$
\phi(\mathcal{C}, \mathcal{D}):=\sup _{\substack{C \in \mathcal{C}, D \in \mathcal{D} \\ \mathbb{P}(C)>0}}|\mathbb{P}(D \mid C)-\mathbb{P}(D)| \quad \text { and } \quad \psi(\mathcal{C}, \mathcal{D}):=\sup _{\substack{C \in \mathcal{C}, D \in \mathcal{D} \\ \mathbb{P}(C), \mathbb{P}(D)>0}}\left|\frac{\mathbb{P}(C \cap D)}{\mathbb{P}(C) \cdot \mathbb{P}(D)}-1\right|
$$

Let $\left(X_{n}\right)_{n \in \mathbb{N}}$ be a (not necessarily stationary) sequence of random variables. For $0 \leq J \leq L \leq \infty$ we can define a $\sigma$-field by

$$
\mathcal{A}_{J}^{L}:=\sigma\left(X_{k}, k \in \mathbb{N} \cap[J, L]\right) .
$$

With that the dependence coefficients are defined by

$$
\phi(n):=\sup _{k \in \mathbb{Z}} \phi\left(\mathcal{A}_{-\infty}^{k}, \mathcal{A}_{k+n}^{\infty}\right) \quad \text { and } \quad \psi(n):=\sup _{k \in \mathbb{Z}} \psi\left(\mathcal{A}_{-\infty}^{k}, \mathcal{A}_{k+n}^{\infty}\right) .
$$

The sequence $\left(X_{n}\right)$ is said to be $\phi$-mixing or $\psi$-mixing if $\phi(n) \rightarrow 0$ or $\psi(n) \rightarrow 0$ as $n \rightarrow \infty$.

It follows easily that

$$
\phi(n) \leq \frac{1}{2} \psi(n)
$$

for all $n \in \mathbb{N}$, see also [Bra05, (1.11)]. For more details about mixing conditions see Bra05].

Now we collect the necessary mixing properties of the continued fractions digits. We start by stating the following lemma from [IK09, Chapter 2.3.4].

Lemma 2.2. Let $\psi=\psi_{\mathfrak{m}}$ denote the $\psi$-mixing coefficient with respect to the continued fraction digits and the Gauss measure $\mathfrak{m}$. Then we have that

$$
\psi_{\mathfrak{m}}(n) \leq \rho \theta^{n-2} \text { for } n \geq 2,
$$

where $\rho=\pi^{2} \log 2 / 6-1$ and $\theta$ is a constant less than 0.30367 , and $\psi_{\mathfrak{m}}(1)=2 \log 2-1$, i.e. the digits of the continued fraction expansion are exponentially $\psi$-mixing.

Next we state the exact value of the $\phi$-mixing coefficient.

Lemma 2.3. Let $\phi=\phi_{\mathfrak{m}}$ denote the $\phi$-mixing coefficient for the Gauss system. Then we have that

$$
\phi_{\mathfrak{m}}(1)=-\frac{1-\log 2+\log \log 2}{\log 2}<0.0861 .
$$

This lemma improves a result of Philipp Phi88, Lemma 2.1] who showed that $\phi_{\mathfrak{m}}(1)<0.4$. We also remark here that the value of $\phi_{\mathfrak{m}}(1)$ coincides with the Erdös-Ford-Tenenbaum constant, see for example For08.

The remaining part of this section is devoted to the proof of Lemma 2.3. One main ingredient for this is the natural extension of the Gauss measure and an associated auxiliary family of measures to be introduced next.

The basic idea is to construct a doubly infinite version of $\left(a_{n}\right)_{n \in \mathbb{N}}$ under $\mathfrak{m}$ for which we use the natural extension. We first define $\bar{G}: I^{2} \rightarrow I^{2}$ by

$$
\bar{G}(\omega, \theta):=\left(G(\omega), \frac{1}{a_{1}(\omega)+\theta}\right) .
$$


It can be easily seen that

$$
\bar{G}^{n}(\omega, \theta)=\left(G^{n}(\omega),\left[a_{n}(\omega), \ldots, a_{2}(\omega), a_{1}(\omega)+\theta\right]\right), n \in \mathbb{N} .
$$

Inhere we slightly abuse notation since $a_{1}(\omega)+\theta$ is usually not a natural number. Then we define the bi-infinite sequence $\left(\bar{a}_{k}\right)_{k \in \mathbb{Z}}$, where each $\bar{a}_{k}: I^{2} \rightarrow \mathbb{N}$ is given by

$$
\bar{a}_{k}(\omega, \theta):=\bar{a}_{1}\left(\bar{G}^{k}(\omega, \theta)\right)
$$

with $\bar{a}_{1}(\omega, \theta):=a_{1}(\omega)=\lfloor 1 / \omega\rfloor$.

Furthermore, we define the extended Gauss measure $\overline{\mathfrak{m}}$ for $B \in \mathcal{B}_{I^{2}}$ by

$$
\overline{\mathfrak{m}}(B):=\frac{1}{\log 2} \cdot \iint_{B} \frac{1}{(x y+1)^{2}} \mathrm{~d} x \mathrm{~d} y,
$$

which is $\bar{G}$ invariant, see for example [IK09, Theorem 1.3.4]. In the following we give one lemma concerning the conditional distribution which are essential in the proof of Lemma 2.3,

Lemma 2.4 (Theorem 1.3.5 of [K09]). For any $v, x \in I$ we have for the conditional probability

$$
\overline{\mathfrak{m}}\left((\omega, \theta) \in[0, x] \times I \mid\left(\bar{a}_{0}(\omega, \theta)=a_{1}(v), \bar{a}_{-1}(\omega, \theta)=a_{2}(v), \ldots\right)\right)=\frac{(v+1) x}{v x+1} \overline{\mathfrak{m}}-a . s .
$$

Motivated by this lemma we also define the probability measure $\mathfrak{m}_{v}$ on $\mathcal{B}_{I}$ via its distribution function, for $v \in I$, by

$$
\mathfrak{m}_{v}([0, x]):=\frac{(v+1) x}{v x+1} .
$$

For further investigations of the natural extension of $\left(a_{n}\right)$ see [K09, Section 1.3].

With this techniques at hand we are now able to begin with the proof of Lemma 2.3 .

Proof of Lemma 2.3. Let $\mathfrak{m}_{v}$ be the measure defined in (9) and let

$$
\eta:=\sup \left\{\left|\mathfrak{m}_{v}(B)-\mathfrak{m}(B)\right|, v \in I, B \in \mathcal{B}_{I}\right\} .
$$

The proof of the lemma is separated into two parts, namely we show that

(A) $\eta=-(1-\log 2+\log \log 2) / \log 2$ and

(B) $\phi_{\mathfrak{m}}(1)=\eta$.

The proof of (B) is inspired by the proof for the first $\psi$-mixing coefficient in [K09].

ad (A) : Let us define $f: I^{2} \rightarrow \mathbb{R}$ by

$$
f(v, x):=\mathfrak{m}_{v}([0, x])-\mathfrak{m}([0, x])=\frac{(v+1) x}{v x+1}-\frac{\log (x+1)}{\log 2} .
$$

We have that $f(v, \cdot)$ is the distribution function of a signed measure with density $\partial f(v, x) / \partial x$. For each $v \in I$ we obtain that $\sup _{\mathcal{B}_{I}}\left(\mathfrak{m}_{v}(B)-\mathfrak{m}(B)\right)$ will be attained for $B=\{x: \partial f(v, x) / \partial x>0\}$ and $\inf _{\mathcal{B}_{I}} \mathfrak{m}_{v}(B)-\mathfrak{m}(B)$ will be attained for $B^{c}$. In the following we will only calculate $\inf _{\mathcal{B}_{I}} \mathfrak{m}_{v}(B)-\mathfrak{m}(B)$ since

$$
\mathfrak{m}_{v}\left(B^{c}\right)-\mathfrak{m}\left(B^{c}\right)=1-\mathfrak{m}_{v}(B)-(1-\mathfrak{m}(B))=-\left(\mathfrak{m}_{v}(B)-\mathfrak{m}(B)\right)
$$

and thus $\sup _{v \in I} \mathfrak{m}_{v}(B)-\mathfrak{m}(B)=-\inf _{v \in I}\left(\mathfrak{m}_{v}(B)-\mathfrak{m}(B)\right)$.

In the next steps we calculate the zeros of $\partial f(v, x) / \partial x$ depending on $v$. We have that

$$
\frac{\partial f(v, x)}{\partial x}=\frac{v+1}{(v x+1)^{2}}-\frac{1}{\log 2 \cdot(x+1)} .
$$

From this we find that the two zeros are given for $v>0$ by

$$
\begin{aligned}
& x_{v, 1}=\frac{(v+1) \cdot \log 2-2 v}{2 v^{2}}+\sqrt{\left(\frac{(v+1) \cdot \log 2-2 v}{2 v^{2}}\right)^{2}-\frac{1-(v+1) \cdot \log 2}{v^{2}}} \text { and } \\
& x_{v, 2}=\frac{(v+1) \cdot \log 2-2 v}{2 v^{2}}-\sqrt{\left(\frac{(v+1) \cdot \log 2-2 v}{2 v^{2}}\right)^{2}-\frac{1-(v+1) \cdot \log 2}{v^{2}}} .
\end{aligned}
$$


If $v=0$ we have that $\partial f(v, x) / \partial x=1-1 /(\log 2 \cdot(x+1))$ and we obtain that $x_{0,2}=1 / \log 2-1$. (Taking limits for $x_{v, 1}$ would lead to the degenerate value of $x_{0,1}=\infty$.)

We obtain as values of interest

$$
x_{2 \log 2-1,1}=1, \quad x_{1,1}=2 \log 2-1, \quad x_{1 / \log 2-1,2}=0, \quad x_{0,2}=1 / \log 2-1 .
$$

In the next steps we will show that $x_{v, 1} \in[0,1]$ if and only if $v \in[2 \log 2-1,1]$ and $x_{v, 2} \in[0,1]$ if and only if $v \in[0,1 / \log 2-1]$ using monotonicity.

We set $g(v):=((v+1) \cdot \log 2-2 v) / 2 v^{2}$ and $h(v):=(1-(v+1) \cdot \log 2) / v^{2}$ such that

$$
x_{v, 1}=g(v)+\sqrt{g(v)^{2}-h(v)} \quad \text { and } \quad x_{v, 2}=g(v)-\sqrt{g(v)^{2}-h(v)} .
$$

We have that

$$
\frac{\partial g(v)}{\partial v}=\frac{2-\log 2}{2 v^{2}}-\frac{\log 2}{v^{3}} \text { and } \frac{\partial h(v)}{\partial v}=\frac{\log 2}{v^{2}}-\frac{2-2 \log 2}{v^{3}} .
$$

First we find that $\partial g(v) / \partial v<0$ and $\partial h(v) / \partial v<0$ for all $v \in I$. To consider $x_{v, 1}$ we notice that $x_{v, 1} \geq 1$ if $g(v) \geq 1$ and in this case it has to hold that $v<2 \log 2-1$. Let us now consider $g(v)<1$. We will show that in this case $x_{v, 1}$ is monotonically decreasing in $v$. Since we have shown that $g$ is monotonically decreasing we only have to show that $\sqrt{g^{2}-h}$ is monotonically decreasing which by the strict monotonicity of the square root is equivalent to the statement that $\partial g^{2}(v) / \partial v-\partial h(v) / \partial v<0$. By applying the chain rule for derivatives this is equivalent to $2 \cdot g(v) \cdot \partial g(v) / \partial v<\partial h(v) / \partial v$. Since we are assuming that $g(v)<1$ this already holds if $2 \cdot \partial g(v) / \partial v<\partial h(v) / \partial v$. Using the derivatives in (10) and noticing that $2-\log 2<2 \log 2$ and $\log 2>2-2 \log 2$ yields that the last statement indeed is true.

In the next steps we consider $x_{v, 2}$. If $g(v)<0$, it immediately follows that $x_{v, 2} \leq 0$ and by the fact that $g$ is monotonically decreasing this only happens for $v>1 / \log 2-1$. Let us now assume that $g(v)<0$. $x_{v, 2}$ is monotonically decreasing if and only if $\partial g(v) / \partial v<\partial \sqrt{g^{2}(v)-h(v)} / \partial v$ which under the assumption that $g(v)<0$ is equivalent to $\partial \sqrt{g^{2}(v)} / \partial v<\partial \sqrt{g^{2}(v)-h(v)} / \partial v$. Since the square root is a strictly monotonic function this is equivalent to $\partial g^{2}(v) / \partial v<\partial\left(g^{2}(v)-h(v)\right) / \partial v$ and thus $\partial h(v) / \partial v<0$ which by our previous notice holds true.

We have that $\partial f(v, x) / \partial x$ changes sign from plus to minus in $x=x_{v, 1}$ for $v \in[2 \log 2-1,1]$ and $\partial f(v, x) / \partial x$ changes sign from minus to plus in $x=x_{v, 2}$ for $v \in[0,1 / \log 2-1]$.

We consider in the following three cases, namely

(a) $0 \leq v<2 \log 2-1$,

(b) $2 \log 2-1 \leq v \leq 1 / \log 2-1$, and

(c) $1 / \log 2-1<v \leq 1$.

$a d$ (国): In this case we have that $\inf _{B \in \mathcal{B}_{I}} \mathfrak{m}_{v}(B)-\mathfrak{m}(B)$ will be attained for $B=\left[0, x_{v, 2}\right]$.

By determining the partial derivative of $f$ with respect to $v$

$$
\frac{\partial f(v, x)}{\partial v}=\frac{x \cdot(1-x)}{(v x+1)^{2}}
$$

we obtain that for all $x \in I$ we have that $\partial f(v, x) / \partial v \geq 0$, i.e. for all $x \in I, f$ is monotonically increasing in $v$. By the fact that $x_{v, 2}$ is monotonically decreasing in $v$ on the relevant parts we obtain for $v>w$ that

$$
\mathfrak{m}_{v}\left(\left[0, x_{v, 2}\right]\right)-\mathfrak{m}\left(\left[0, x_{v, 2}\right]\right)=f\left(v, x_{v, 2}\right) \geq f\left(w, x_{v, 2}\right) \geq f\left(w, x_{w, 2}\right) .
$$

Thus, $\inf _{v \in[0,2 \log 2-1), B \in \mathcal{B}_{I}} \mathfrak{m}_{v}(B)-\mathfrak{m}(B)=\inf _{B \in \mathcal{B}_{I}} \mathfrak{m}_{0}(B)-\mathfrak{m}(B)$. Using $x_{0,2}=1 / \log 2-1$ we find

$$
f\left(0, \frac{1}{\log 2}-1\right)=\frac{1-\log 2+\log \log 2}{\log 2}
$$

and consequently

$$
\inf _{v \in[0,2 \log 2-1), B \in \mathcal{B}_{I}} \mathfrak{m}_{v}(B)-\mathfrak{m}(B)=\frac{1-\log 2+\log \log 2}{\log 2}
$$


ad (B): In this case we have that $\inf _{B \in \mathcal{B}_{I}}\left(\mathfrak{m}_{v}(B)-\mathfrak{m}(B)\right)$ will be attained for $B=\left[0, x_{v, 2}\right) \cup\left[x_{v, 1}, 1\right)$. Furthermore, the monotonicity of $f$ in $v$ and a similar argument as in (11) yields

$$
\begin{aligned}
& \quad \inf _{v \in[2 \log 2-1,1 / \log 2-1], B \in \mathcal{B}_{I}} \mathfrak{m}_{v}(B)-\mathfrak{m}(B) \\
& \geq \inf _{v \in[2 \log 2-1,1 / \log 2-1]} \mathfrak{m}_{v}\left(\left[0, x_{v, 2}\right)\right)-\mathfrak{m}\left(\left[0, x_{v, 2}\right)\right) \\
& \quad+\quad \inf _{w \in[2 \log 2-1,1 / \log 2-1]} \mathfrak{m}_{w}\left(\left[x_{w, 1}, 1\right)\right)-\mathfrak{m}\left(\left[x_{w, 1}, 1\right)\right) \\
& =\inf _{v \in[2 \log 2-1,1 / \log 2-1]} f\left(v, x_{v, 2}\right)+\inf _{w \in[2 \log 2-1,1 / \log 2-1]}-f\left(w, x_{w, 1}\right) .
\end{aligned}
$$

For the first summand in (13) we have by the monotonicity of $f$ in the first argument that the infimum will be attained for $v=2 \log 2-1$ in $x_{2} \log 2-1,2$ and for the second summand in (13) we again have by the monotonicity of $f$ in the first argument that the infimum will be attained for $v=1 / \log 2-1$ in $x_{1 / \log 2-1,1}$, i.e. we have

$$
\begin{aligned}
\inf _{v \in[2 \log 2-1,1 / \log 2-1]} f\left(v, x_{v, 2}\right) & =f\left(2 \log 2-1, x_{2} \log 2-1,2\right) \text { and } \\
\inf _{w \in[2 \log 2-1,1 / \log 2-1]}-f\left(w, x_{w, 1}\right) & =-f\left(1 / \log 2-1, x_{1 / \log 2-1,1}\right)=f\left(2 \log 2-1, x_{2} \log 2-1,2\right) .
\end{aligned}
$$

Combining (13) with (14) and (15) yields

$$
\inf _{v \in[2 \log 2-1,1 / \log 2-1], B \in \mathcal{B}_{I}} \mathfrak{m}_{v}(B)-\mathfrak{m}(B) \geq 2 \cdot f\left(2 \log 2-1, x_{2} \log 2-1,2\right) \geq-0.0118 .
$$

$a d$ (ㅈ): In this case we have that $\inf _{B \in \mathcal{B}_{I}}\left(\mathfrak{m}_{v}(B)-\mathfrak{m}(B)\right)$ will be attained for $B=\left[x_{v, 1}, 1\right)$. Furthermore, since $f(v, 1)=0$, and by the monotonicity of $f$, using the argument as in (11) in the reverse direction, we have that

$$
\begin{aligned}
\inf _{v \in(1 / \log 2-1,1)} \mathfrak{m}_{v}\left(\left[x_{v, 1}, 1\right)\right)-\mathfrak{m}\left(\left[x_{v, 1}, 1\right]\right) & =\inf _{v \in(1 / \log 2-1, v]}-f\left(v, x_{v, 1}\right) \\
& =-f\left(1, x_{1,1}\right) \\
& =-f(1,2 \log 2-1) \\
& =\frac{1-\log 2+\log \log 2}{\log 2} .
\end{aligned}
$$

Putting (12), (16), and (17) together and noticing that $-0.0118>(1-\log 2+\log \log 2) / \log 2$ yields the first statement.

ad $(\mathbb{B})$ : To prove the second part of the lemma we make use of the natural extension of the Gauss system. We have that

$$
\phi_{\mathfrak{m}}(1)=\sup \left\{|\overline{\mathfrak{m}}(\bar{B} \mid \bar{A})-\overline{\mathfrak{m}}(\bar{B})|: \bar{B} \in \sigma\left(\bar{a}_{n}, \bar{a}_{n+1}, \ldots\right), \bar{A} \in \sigma\left(\ldots, \bar{a}_{n-2}, \bar{a}_{n-1}\right), \overline{\mathfrak{m}}(\bar{A})>0, n \in \mathbb{N}\right\} .
$$

This follows directly from the definition of the bi-infinite sequence $\left(\bar{a}_{n}\right)_{n \in \mathbb{Z}}$ and the definition of the $\phi$-mixing coefficients. By the shift invariance of $\mathfrak{m}$ the supremum does not change if we restrict $n$ to 1 and we thus only consider $\bar{B} \in \sigma\left(\bar{a}_{1}, \bar{a}_{2}, \ldots\right)$ and $\bar{A} \in \sigma\left(\bar{a}_{0}, \bar{a}_{-1}, \ldots\right)$ for which $\overline{\mathfrak{m}}(\bar{A})>0$.

Clearly, $\bar{B}=B \times I$ and $\bar{A}=I \times A$, for some $A, B \in \mathcal{B}_{I}$. Thus,

$$
\phi_{\mathfrak{m}}(1)=\sup \left\{\left|\frac{\overline{\mathfrak{m}}(A \times B)}{\mathfrak{m}(A)}-\mathfrak{m}(B)\right|: A, B \in \mathcal{B}_{I}, \mathfrak{m}(A)>0\right\} .
$$

Furthermore, we have that $\overline{\mathfrak{m}}(A \times B)=\int_{A} \mathfrak{m}_{v}(B) \mathrm{d} \mathfrak{m}(v)$, for $A, B \in \mathcal{B}_{I}$. For given $B \in \mathcal{B}_{I}$ we have that

$$
\sup _{v \in I} \mathfrak{m}_{v}(B) \geq \sup _{A \in \mathcal{B}_{I}} \frac{\int_{A} \mathfrak{m}_{v}(B) \mathrm{d} \mathfrak{m}(v)}{\mathfrak{m}(A)} \text { and } \inf _{v \in I} \mathfrak{m}_{v}(B) \leq \inf _{A \in \mathcal{B}_{I}} \frac{\int_{A} \mathfrak{m}_{v}(B) \mathrm{d} \mathfrak{m}(v)}{\mathfrak{m}(A)} .
$$

In the next steps we show that we have equality if $\mathfrak{m}_{v}(B)$ is continuous in $v$ for given $B$. In this case the supremum is attained on $[0,1]$, say on a point $x_{B}$ and we have that

$$
\sup _{A \in \mathcal{B}_{I}} \frac{\int_{A} \mathfrak{m}_{v}(B) \mathrm{d} \mathfrak{m}(v)}{\mathfrak{m}(A)}=\lim _{\epsilon \searrow 0} \frac{\int_{\left[x_{B}-\epsilon, x_{B}+\epsilon\right] \cap[0,1]} \mathfrak{m}_{v}(B) \mathrm{d} \mathfrak{m}(v)}{\mathfrak{m}\left(\left[x_{B}-\epsilon, x_{B}+\epsilon\right] \cap[0,1]\right)}=\sup _{v \in I} \mathfrak{m}_{v}(B)
$$


and analogously for the infimum. On the other hand we have by our calculation from (A) that

$$
\begin{aligned}
\sup \left\{\left|\mathfrak{m}_{v}(B)-\mathfrak{m}(B)\right|, v \in I, B \in \mathcal{B}_{I}\right\} & =\left|\mathfrak{m}_{0}([0,1-1 / \log 2-1])-\mathfrak{m}([0,1-1 / \log 2-1])\right| \\
& =|f(0,[0,1 / \log 2-1])|
\end{aligned}
$$

and $f(v,[0,1 / \log 2-1])$ is continuous in $v$. Now the claim in (B) follows from this argument, (18) and the definition of $\eta$ by noting that

$$
\begin{aligned}
\phi_{\mathfrak{m}}(1) & =\sup \left\{\left|\frac{\int_{A} \mathfrak{m}_{v}(B) \mathrm{d} \mathfrak{m}(v)}{\mathfrak{m}(A)}-\mathfrak{m}(B)\right|: A, B \in \mathcal{B}_{I}, \mathfrak{m}(A)>0\right\} \\
& =\sup \left\{\left|\mathfrak{m}_{v}(B)-\mathfrak{m}(B)\right|: v \in I, B \in \mathcal{B}_{I}\right\}=\eta
\end{aligned}
$$

\section{Proofs of the Zero-one LAWS}

All the zero-one laws can be proven by the following lemma which is a simplified version of Phi67, Theorem 3].

Lemma 3.1 ( [Phi67, Theorem 3]). Let $\left(\Gamma_{n}\right)_{n \in \mathbb{N}}$ be a sequence of measurable sets in a probability space $(\Omega, \mathcal{A}, \mu)$. Suppose that there exists a function $q: \mathbb{N} \rightarrow \mathbb{R}_{\geq 0}$ fulfilling $\sum_{n=1}^{\infty} q(n)<\infty$ such that for all integers $n>m$ we have

$$
\mu\left(\Gamma_{m} \cap \Gamma_{n}\right) \leq \mu\left(\Gamma_{m}\right) \cdot \mu\left(\Gamma_{n}\right)+q(n-m) \cdot \mu\left(\Gamma_{n}\right) .
$$

Then $\Gamma_{n}$ holds infinitely often with Lebesgue measure 0 or 1 according as $\sum_{n=1}^{\infty} \mu\left(\Gamma_{n}\right)$ is finite or not.

Using Lemma 2.2 we immediately obtain the following lemma, which enables us to prove Theorem 1.6 similarly as in the i.i.d. setting.

Lemma 3.2. Let $\left(A_{n}\right)$ be a sequence of events such that $A_{n} \in \sigma\left(a_{n}\right)$ for all $n \in \mathbb{N}$. If $\sum_{n \in \mathbb{N}} \mathfrak{m}\left(A_{n}\right)=\infty$, then $\mathfrak{m}\left(\limsup _{n \rightarrow \infty} A_{n}\right)=1$.

Proof of Theorem 1.6. Since $x \cdot \log (2) \leq \log (1+x) \leq x$ for all $x \in[0,1)$, it follows that for all considered sets $A_{n}$

$$
\frac{\lambda\left(A_{n}\right)}{\log 2} \leq \mathfrak{m}\left(A_{n}\right) \leq \frac{2 \cdot \lambda\left(A_{n}\right)}{\log 2} .
$$

Hence, it suffices to determine a condition for $\sum_{n=1}^{\infty} \lambda\left(A_{n}\right)=\infty$. We first prove (B) as (A) follows from (B) by setting for example $c_{n}=2 d_{n}$.

ad (B): We have that

$$
\lambda\left(A_{n}\right)=\frac{1}{d_{n}}-\frac{1}{d_{n}+\left\lfloor d_{n} / c_{n}\right\rfloor+1} .
$$

An easy calculation shows that

$$
\lambda\left(A_{n}\right) \leq \frac{1}{d_{n}}-\frac{1}{d_{n}+d_{n} / c_{n}+1}<\frac{1}{c_{n} d_{n}}+\frac{1}{d_{n}^{2}} .
$$

Hence, if $\Gamma$ is finite, the first Borel-Cantelli implies $\lambda\left(\lim \sup _{n \rightarrow \infty} A_{n}\right)=0$.

To prove the second part we first notice that

$$
\lambda\left(A_{n}\right)>\frac{1}{d_{n}}-\frac{1}{d_{n}+d_{n} / c_{n}}>\frac{1}{2 c_{n} d_{n}},
$$

i.e. $\sum_{n \in \mathbb{N}} \lambda\left(A_{n}\right)$ diverges if $\sum_{n \in \mathbb{N}} 1 /\left(c_{n} d_{n}\right)$ does. Next we assume that $\sum_{n \in \mathbb{N}} 1 / d_{n}^{2}=\infty$. Clearly, for all $n \in \mathbb{N}$, we have

and thus

$$
\left\{a_{n}=d_{n}\right\} \subset\left\{d_{n} \leq a_{n} \leq d_{n}+\frac{d_{n}}{c_{n}}\right\}
$$

$$
\lambda\left(A_{n}\right) \geq \lambda\left(a_{n}=d_{n}\right)=\frac{1}{d_{n}}-\frac{1}{d_{n}+1} \geq \frac{1}{2 d_{n}^{2}},
$$

i.e. $\sum_{n=1}^{\infty} \lambda\left(A_{n}\right)$ diverges if $\sum_{n=1}^{\infty} 1 / d_{n}^{2}$ does. Since $A_{n} \in \sigma\left(a_{n}\right)$, we can apply Lemma 3.2 and obtain the statement of (B). 
ad (C): We notice that

$$
\lambda\left(A_{n}\right)=\frac{1}{d_{n}+1}-\frac{1}{d_{n}+\left\lfloor d_{n} / c_{n}\right\rfloor+1} .
$$

An easy calculations shows that

$$
\lambda\left(A_{n}\right) \leq \begin{cases}0 & \text { if } c_{n}>d_{n} \\ \frac{1}{d_{n} c_{n}} & \text { else }\end{cases}
$$

and by the first Borel-Cantelli Lemma $\sum_{n: c_{n} \leq d_{n}} 1 /\left(c_{n} d_{n}\right)<\infty \operatorname{implies} \lambda\left(\limsup _{n \rightarrow \infty} A_{n}\right)=0$.

For proving the second part we make use of an analogous statement as in the proof of $(\mathbb{B})$. With $A_{n} \in \sigma\left(a_{n}\right)$ we have $\lambda\left(A_{n}\right)>0$ if and only if $c_{n} \leq d_{n}$. Hence, we are left to show that $\sum_{n \in \mathbb{N}} \lambda\left(A_{n}\right)$ diverges if $\sum_{n: c_{n} \leq d_{n}} 1 /\left(c_{n} d_{n}\right)$ does.

First, let us assume that $\left\lfloor d_{n} / c_{n}\right\rfloor=1$. Then $\lambda\left(A_{n}\right)=\lambda\left(a_{n}=d_{n}\right)>1 /\left(2 d_{n}^{2}\right) \geq 1 /\left(2 c_{n} d_{n}\right)$ which follows from (21) and the restriction $\left\lfloor d_{n} / c_{n}\right\rfloor=1$.

Next, we assume that $d_{n} / c_{n} \geq 2$. This assumptions yields

$$
\lambda\left(A_{n}\right)>\frac{1}{d_{n}+1}-\frac{1}{d_{n}+d_{n} / c_{n}}>\frac{d_{n}-c_{n}}{4 c_{n} d_{n}^{2}} \geq \frac{1}{8 c_{n} d_{n}} .
$$

Lemma 3.2 gives the statement of $[$ C).

The proof of Theorem 1.11 needs some extra attention and follows as a corollary of Theorem 1.6.

Proof of Theorem 1.11. We first define

$$
c_{n}^{\prime}:=\frac{c_{n} d_{n}}{d_{n}+3 c_{n}} \text { and } c_{n}^{\prime \prime}:=\frac{c_{n} d_{n}}{d_{n}+4 c_{n}} .
$$

Assume for the following that $\sum_{n \in \mathbb{N}} 1 /\left(c_{n} d_{n}\right)<\infty$. If $c_{n} \leq d_{n} /(3+\epsilon)$ we have that

$$
\sum_{n \in \mathbb{N}} \frac{1}{c_{n}^{\prime}\left\lfloor d_{n}\right\rfloor}=\sum_{n \in \mathbb{N}} \frac{1}{\left\lfloor d_{n}\right\rfloor} \cdot \frac{d_{n}+3 c_{n}}{c_{n} d_{n}} \leq \sum_{n \in \mathbb{N}} \frac{1}{\left\lfloor d_{n}\right\rfloor} \cdot \frac{2 d_{n}}{c_{n} d_{n}}=\sum_{n \in \mathbb{N}} \frac{2}{c_{n}\left\lfloor d_{n}\right\rfloor}=\infty .
$$

Similarly, we obtain if $c_{n} \leq d_{n} /(4+\epsilon)$ that

$$
\sum_{n \in \mathbb{N}} \frac{1}{c_{n}^{\prime \prime}\left\lfloor d_{n}\right\rfloor}=\sum_{n \in \mathbb{N}} \frac{1}{\left\lfloor d_{n}\right\rfloor} \cdot \frac{d_{n}+4 c_{n}}{c_{n} d_{n}} \leq \sum_{n \in \mathbb{N}} \frac{1}{\left\lfloor d_{n}\right\rfloor} \cdot \frac{2 d_{n}}{c_{n} d_{n}}=\sum_{n \in \mathbb{N}} \frac{2}{c_{n}\left\lfloor d_{n}\right\rfloor}=\infty .
$$

The assumptions $c_{n} \leq d_{n} /(3+\epsilon)$ or $c_{n} \leq d_{n} /(4+\epsilon)$ respectively and $\sum_{n \in \mathbb{N}} 1 /\left(c_{n} d_{n}\right)<\infty$ imply further that $\sum_{n \in \mathbb{N}} 1 /\left\lfloor d_{n}\right\rfloor^{2}<\infty$. The second statement of Theorem [1.6 implies then that

$$
\lambda\left(\limsup _{n \rightarrow \infty}\left\{\left\lfloor d_{n}\right\rfloor \leq a_{n} \leq\left\lfloor d_{n}\right\rfloor\left(1+\frac{1}{c_{n}^{\prime}}\right)\right\}\right)=0 \text { or } \lambda\left(\limsup _{n \rightarrow \infty}\left\{\left\lfloor d_{n}\right\rfloor \leq a_{n} \leq\left\lfloor d_{n}\right\rfloor\left(1+\frac{1}{c_{n}^{\prime \prime}}\right)\right\}\right)=0
$$

respectively. By the definition of $c_{n}^{\prime}$ and $c_{n}^{\prime \prime}$ this implies that

$$
\begin{aligned}
& \lambda\left(\limsup _{n \rightarrow \infty}\left\{\left\lfloor d_{n}\right\rfloor \leq a_{n} \leq\left\lfloor d_{n}\right\rfloor\left(1+\frac{1}{c_{n}}\right)+3\right\}\right)=0 \text { or } \\
& \lambda\left(\limsup _{n \rightarrow \infty}\left\{\left\lfloor d_{n}\right\rfloor \leq a_{n} \leq\left\lfloor d_{n}\right\rfloor\left(1+\frac{1}{c_{n}}\right)+4\right\}\right)=0
\end{aligned}
$$

respectively. From (A) and (B) of Lemma 1.10 we can conclude that

$$
\left\{d_{n} \leq r_{n}, y_{n} \leq d_{n}\left(1+\frac{1}{c_{n}}\right)\right\} \subset\left\{d_{n} \leq a_{n} \leq d_{n}\left(1+\frac{1}{c_{n}}\right)+1\right\} \subset\left\{\left\lfloor d_{n}\right\rfloor \leq a_{n} \leq\left\lfloor d_{n}\right\rfloor\left(1+\frac{1}{c_{n}}\right)+3\right\}
$$

and from (C) of Lemma 1.10 we can conclude that

$$
\left\{d_{n} \leq u_{n} \leq d_{n}\left(1+\frac{1}{c_{n}}\right)\right\} \subset\left\{d_{n} \leq a_{n} \leq d_{n}\left(1+\frac{1}{c_{n}}\right)+2\right\} \subset\left\{\left\lfloor d_{n}\right\rfloor \leq a_{n} \leq\left\lfloor d_{n}\right\rfloor\left(1+\frac{1}{c_{n}}\right)+4\right\} .
$$

Hence, (23) or (24) respectively imply

$$
\lambda\left(\limsup _{n \rightarrow \infty}\left\{d_{n} \leq z_{n} \leq d_{n} \cdot\left(1+\frac{1}{c_{n}}\right)\right\}\right)=0,
$$

where $\left(z_{n}\right)=\left(r_{n}\right),\left(z_{n}\right)=\left(y_{n}\right)$, or $\left(z_{n}\right)=\left(u_{n}\right)$. 
In order to prove the infinite part we first define

$$
\bar{c}_{n}^{\prime}:=\frac{c_{n} d_{n}}{d_{n}-3 c_{n}} \text { and } \bar{c}_{n}^{\prime \prime}:=\frac{c_{n} d_{n}}{d_{n}-4 c_{n}}
$$

and assume for the following that $\sum_{n \in \mathbb{N}} 1 /\left(c_{n} d_{n}\right)=\infty$. If $c_{n} \leq d_{n} /(3+\epsilon)$, we have that

$$
\sum_{n \in \mathbb{N}} \frac{1}{\bar{c}_{n}^{\prime}\left\lceil d_{n}\right\rceil}=\sum_{n \in \mathbb{N}} \frac{1}{\left\lceil d_{n}\right\rceil} \cdot \frac{d_{n}-3 c_{n}}{c_{n} d_{n}} \geq \sum_{n \in \mathbb{N}} \frac{1}{\left\lceil d_{n}\right\rceil} \cdot \frac{\epsilon}{3+\epsilon} \cdot \frac{d_{n}}{c_{n} d_{n}}=\sum_{n \in \mathbb{N}} \frac{\epsilon}{3+\epsilon} \cdot \frac{1}{c_{n}\left\lceil d_{n}\right\rceil}=\infty .
$$

Similarly, we obtain if $c_{n} \leq d_{n} /(4+\epsilon)$ that

$$
\sum_{n \in \mathbb{N}} \frac{1}{\bar{c}_{n}^{\prime \prime}\left\lceil d_{n}\right\rceil}=\sum_{n \in \mathbb{N}} \frac{1}{\left\lceil d_{n}\right\rceil} \cdot \frac{d_{n}-4 c_{n}}{c_{n} d_{n}} \geq \sum_{n \in \mathbb{N}} \frac{1}{\left\lceil d_{n}\right\rceil} \cdot \frac{\epsilon}{4+\epsilon} \cdot \frac{d_{n}}{4 c_{n} d_{n}}=\sum_{n \in \mathbb{N}} \frac{\epsilon}{4+\epsilon} \cdot \frac{1}{c_{n}\left\lceil d_{n}\right\rceil}=\infty .
$$

Applying the other direction of Theorem 1.6 yields then under the assumption that $c_{n} \leq d_{n} /(3+\epsilon)$ or $c_{n} \leq d_{n} /(4+\epsilon)$ respectively that

$$
\lambda\left(\limsup _{n \rightarrow \infty}\left\{\left\lceil d_{n}\right\rceil \leq a_{n} \leq\left\lceil d_{n}\right\rceil\left(1+\frac{1}{\bar{c}_{n}^{\prime}}\right)\right\}\right)=1 \text { or } \lambda\left(\limsup _{n \rightarrow \infty}\left\{\left\lceil d_{n}\right\rceil \leq a_{n} \leq\left\lceil d_{n}\right\rceil\left(1+\frac{1}{\bar{c}_{n}^{\prime \prime}}\right)\right\}\right)=1
$$

respectively. By the definition of $\bar{c}_{n}^{\prime}$ and $\bar{c}_{n}^{\prime \prime}$ this implies that

$$
\begin{aligned}
& \lambda\left(\limsup _{n \rightarrow \infty}\left\{\left\lceil d_{n}\right\rceil \leq a_{n} \leq\left\lceil d_{n}\right\rceil\left(1+\frac{1}{c_{n}}\right)-3\right\}\right)=1 \text { or } \\
& \lambda\left(\limsup _{n \rightarrow \infty}\left\{\left\lceil d_{n}\right\rceil \leq a_{n} \leq\left\lceil d_{n}\right\rceil\left(1+\frac{1}{c_{n}}\right)-4\right\}\right)=1
\end{aligned}
$$

respectively. From (A) and (B) of Lemma 1.10 we know that

$\left\{d_{n} \leq r_{n}, y_{n} \leq d_{n}\left(1+\frac{1}{c_{n}}\right)\right\} \supset\left\{d_{n} \leq a_{n} \leq d_{n}\left(1+\frac{1}{c_{n}}\right)-1\right\} \supset\left\{\left\lceil d_{n}\right\rceil \leq a_{n} \leq\left\lceil d_{n}\right\rceil\left(1+\frac{1}{c_{n}}\right)-3\right\}$ and from $(\mathrm{Cl})$ of Lemma 1.10 we know that

$$
\left\{d_{n} \leq u_{n} \leq d_{n}\left(1+\frac{1}{c_{n}}\right)\right\} \supset\left\{d_{n} \leq a_{n} \leq d_{n}\left(1+\frac{1}{c_{n}}\right)-2\right\} \supset\left\{\left\lceil d_{n}\right\rceil \leq a_{n} \leq\left\lceil d_{n}\right\rceil\left(1+\frac{1}{c_{n}}\right)-4\right\} .
$$

Hence, (25) or (26) respectively imply

$$
\lambda\left(\limsup _{n \rightarrow \infty}\left\{d_{n} \leq z_{n} \leq d_{n}\left(1+\frac{1}{c_{n}}\right)\right\}\right)=1,
$$

where $\left(z_{n}\right)=\left(r_{n}\right),\left(z_{n}\right)=\left(y_{n}\right)$, or $\left(z_{n}\right)=\left(u_{n}\right)$.

\section{Proof of the central limit theorems}

We will first prove Theorem 1.3 and show afterwards that Theorem 1.4 and Theorem 1.5 can be considered as special cases of this theorem. To prove Theorem 1.3 we will use the following lemma which is a special form of [Phi70, Theorem 3]. For the following we set $v_{n} \ll w_{n}$ if there exists a constant $K>0$ such that $v_{n} \leq K \cdot w_{n}$ for all $n \in \mathbb{N}$.

Lemma 4.1. Let $\left(x_{n}\right)$ be a sequence of centered random variables with $\sup _{n \in \mathbb{N}}\left\|x_{n}\right\|_{\infty} \leq 1$ and

$$
s_{n}^{2}:=\mathbb{E}\left(\left(\sum_{k=1}^{n} x_{k}\right)^{2}\right) \rightarrow \infty .
$$

Denote by $\mathcal{M}_{a, b}$ the $\sigma$-algebra generated by the events $\left\{x_{n}<z\right\}$ with $z \in \mathbb{R}$ and $1 \leq a \leq n \leq b \leq \infty$. Suppose that there exist $\theta \in(0,1)$ such that for all events $A \in \mathcal{M}_{1, r}$ and $B \in \mathcal{M}_{r+n, \infty}$ we have

$$
\mathbb{P}(A \cap B)-\mathbb{P}(A) \cdot \mathbb{P}(B) \ll \theta^{n} \cdot \mathbb{P}(A) \cdot \mathbb{P}(B) .
$$

Moreover, assume that

$$
\sum_{i=m+1}^{m+n} \mathbb{E}\left(\left|x_{i}\right|\right) \ll \mathbb{E}\left(\left(\sum_{i=m+1}^{m+n} x_{i}\right)^{2}\right),
$$


uniformly in $m$. Then

$$
\lim _{n \rightarrow \infty} \mathbb{P}\left(\frac{\sum_{i=1}^{n} x_{i}}{s_{n}^{2}}<z\right)=\frac{1}{\sqrt{2 \pi}} \int_{-\infty}^{z} e^{-t^{2} / 2} \mathrm{~d} t
$$

Proof of Theorem 1.3. We set $x_{n}=\mathbb{1}_{A_{n}}-\mathbb{E}\left(\mathbb{1}_{A_{n}}\right)$. That condition (27) holds follows from Lemma 2.2. Hence, we are left to show (28), i.e. it suffices to show that there exists $\epsilon^{\prime}>0$ such that for all $m \in \mathbb{N}_{0}$, $n \in \mathbb{N}$

$$
\epsilon^{\prime} \cdot \sum_{i=m+1}^{m+n} \mathbb{V}\left(\mathbb{1}_{A_{i}}\right)<\mathbb{V}\left(\sum_{i=m+1}^{m+n} \mathbb{1}_{A_{i}}\right)
$$

We first notice that

$$
\begin{aligned}
\mathbb{V}\left(\sum_{i=m+1}^{m+n} \mathbb{1}_{A_{i}}\right) & =\sum_{i=m+1}^{m+n}\left(\mathbb{V}\left(\mathbb{1}_{A_{i}}\right)+\sum_{j=i+1}^{m+n} \operatorname{Cov}\left(\mathbb{1}_{A_{i}}, \mathbb{1}_{A_{j}}\right)\right) \\
& \geq \sum_{i=m+1}^{m+n}\left(\mathbb{V}\left(\mathbb{1}_{A_{i}}\right)-\sum_{j>i}^{m+n}\left|\operatorname{Cov}\left(\mathbb{1}_{A_{i}}, \mathbb{1}_{A_{j}}\right)\right|\right) .
\end{aligned}
$$

To estimate the last summands we notice that

$$
\sum_{j=i+1}^{m+n}\left|\operatorname{Cov}\left(\mathbb{1}_{A_{i}}, \mathbb{1}_{A_{j}}\right)\right|=0
$$

if $\mathfrak{m}\left(A_{i}\right)=1$. Assume now that $\mathfrak{m}\left(A_{i}\right)<1$. For $i<j$ we have that

$$
\left|\operatorname{Cov}\left(\mathbb{1}_{A_{i}}, \mathbb{1}_{A_{j}}\right)\right|=\left|\mathfrak{m}\left(A_{i} \cap A_{j}\right)-\mathfrak{m}\left(A_{i}\right) \cdot \mathfrak{m}\left(A_{j}\right)\right| \leq \phi_{\mathfrak{m}}(j-i) \cdot \mathfrak{m}\left(A_{i}\right)
$$

and on the other hand

$$
\left|\operatorname{Cov}\left(\mathbb{1}_{A_{i}}, \mathbb{1}_{A_{j}}\right)\right|=\left|\mathfrak{m}\left(A_{i}^{c} \cap A_{j}\right)-\mathfrak{m}\left(A_{i}^{c}\right) \cdot \mathfrak{m}\left(A_{j}\right)\right| \leq \phi_{\mathfrak{m}}(j-i) \cdot \mathfrak{m}\left(A_{i}^{c}\right) .
$$

Thus, Lemma 2.3 and Lemma 2.2 with an application of (8) give

$$
\begin{aligned}
\sum_{j>i}\left|\operatorname{Cov}\left(\mathbb{1}_{A_{i}}, \mathbb{1}_{A_{j}}\right)\right| & \leq\left(\sum_{n=1}^{\infty} \phi_{\mathfrak{m}}(n)\right) \cdot \min \left\{\mathfrak{m}\left(A_{i}\right), \mathfrak{m}\left(A_{i}^{c}\right)\right\} \\
& \leq\left(\frac{1-\log 2+\log \log 2}{-\log 2}+\left(\frac{\pi^{2} \cdot \log 2}{6}-1\right) \cdot \sum_{n=0}^{\infty} \theta^{n}\right) \cdot \min \left\{\mathfrak{m}\left(A_{i}\right), \mathfrak{m}\left(A_{i}^{c}\right)\right\} \\
& =\kappa \cdot \min \left\{\mathfrak{m}\left(A_{i}\right), \mathfrak{m}\left(A_{i}^{c}\right)\right\}
\end{aligned}
$$

with $\kappa$ being defined as

$$
\kappa:=\frac{\left(\pi^{2} \log 2\right) / 6-1}{1-\theta}-\frac{1-\log 2+\log \log 2}{\log 2}<\frac{1}{2} .
$$

On the other hand we have that

$$
\mathbb{V}\left(\mathbb{1}_{A_{i}}\right)=\mathfrak{m}\left(A_{i}\right) \cdot \mathfrak{m}\left(A_{i}^{c}\right) \geq \frac{\min \left\{\mathfrak{m}\left(A_{i}\right), \mathfrak{m}\left(A_{i}^{c}\right)\right\}}{2} .
$$

Hence, we can conclude from (30) that

$$
\begin{aligned}
\mathbb{V}\left(\sum_{i=m+1}^{m+n} \mathbb{1}_{A_{i}}\right) & >\sum_{i=m+1}^{m+n}\left(\frac{\min \left\{\mathfrak{m}\left(A_{i}\right), \mathfrak{m}\left(A_{i}^{c}\right)\right\}}{2}-\kappa \cdot \min \left\{\mathfrak{m}\left(A_{i}\right), \mathfrak{m}\left(A_{i}^{c}\right)\right\}\right) \\
& =\left(\frac{1}{2}-\kappa\right) \cdot \sum_{i=m+1}^{m+n} \min \left\{\mathfrak{m}\left(A_{i}\right), \mathfrak{m}\left(A_{i}^{c}\right)\right\} \\
& \geq\left(\frac{1}{2}-\kappa\right) \cdot \sum_{i=m+1}^{m+n} \mathbb{V}\left(\mathbb{1}_{A_{i}}\right),
\end{aligned}
$$

proving (29). 
Remark 4.2 For this proof we indeed made use of the precise value of the first $\phi$-mixing coefficient to show that $\mathbb{V}\left(\sum_{i=1}^{n} \mathbb{1}_{A_{i}}\right)$ grows at least proportional to $\sum_{i=1}^{n} \mathbb{V}\left(\mathbb{1}_{A_{i}}\right)$. One can see that the constant $\kappa$ in (33) has to be less than $1 / 2$ for the factor in (34) to be positive.

The crucial point is the estimate in (30). The covariances appearing there will be estimated with the use of the $\phi$-mixing coefficients as in (31) and (32). Using the less precise estimate $\phi_{\mathfrak{m}}(1) \leq \psi(1) / 2=\log 2-1 / 2$ together with the estimates in Lemma 2.2 is not precise enough to conclude $\sum_{n=1}^{\infty} \phi_{\mathfrak{m}}(n)<1 / 2$.

The first $\phi$-mixing coefficient has already been estimated before. In Sam85] Samur showed $\phi_{\mathfrak{m}}(1)<1$, which was later improved by Philipp to $\phi(1) \leq 0.4$ in Phi88, Lemma 2.1]. For this Philipp used a weaker estimate on the first $\psi$-mixing coefficient compared to the one mentioned above. All these previous estimates are not strong enough to imply $\sum_{n=1}^{\infty} \phi_{\mathfrak{m}}(n)<1 / 2$.

Proof of Theorem 1.4. The theorem follows as a direct application of Theorem 1.3. What remains to be shown is that (4) implies $\sum_{n=1}^{\infty} \mathbb{V}\left(\mathbb{1}_{B_{n}}\right)=\infty$ with $B_{n}:=\left\{a_{n}>b_{n}\right\}$. From (19) it follows that

$$
\sum_{n=1}^{\infty} \mathbb{V}\left(\mathbb{1}_{B_{n}}\right) \geq \sum_{n=1}^{\infty} \mathfrak{m}\left(a_{n}=1\right) \cdot \mathfrak{m}\left(B_{n}\right) \geq \frac{\mathfrak{m}\left(a_{1}=1\right)}{\log 2} \cdot \sum_{n=1}^{\infty} \lambda\left(B_{n}\right)=\frac{\mathfrak{m}\left(a_{n}=1\right)}{\log 2} \cdot \sum_{n=1}^{\infty} \frac{1}{b_{n}+1}
$$

and thus (4) implies $\sum_{n=1}^{\infty} \mathbb{V}\left(\mathbb{1}_{B_{n}}\right)=\infty$.

Proof of Theorem 1.5. As in the proof of Theorem 1.4 we only have to prove that the given conditions imply

$$
\sum_{n=1}^{\infty} \mathbb{V}\left(\mathbb{1}_{A_{n}}\right)=\infty
$$

ad (A): We have that

$$
\sum_{n=1}^{\infty} \mathbb{V}\left(\mathbb{1}_{A_{n}}\right) \geq \sum_{n=1}^{\infty} \mathfrak{m}\left(a_{n}=1\right) \cdot \mathfrak{m}\left(A_{n}\right) \geq \frac{\mathfrak{m}\left(a_{1}=1\right)}{\log 2} \cdot \sum_{n=1}^{\infty} \lambda\left(A_{n}\right)=\frac{\mathfrak{m}\left(a_{n}=1\right)}{\log 2} \cdot \sum_{n=1}^{\infty}\left(\frac{1}{d_{n}}-\frac{1}{d_{n}+1}\right)
$$

and thus $\sum_{n=1}^{\infty} 1 / d_{n}^{2}=\infty$ implies $\underline{351}$.

ad (B) : We have that

$$
\sum_{n=1}^{\infty} \mathbb{V}\left(\mathbb{1}_{A_{n}}\right)=\sum_{n: d_{n}=1} \mathbb{V}\left(\mathbb{1}_{A_{n}}\right)+\sum_{n: d_{n}>1} \mathbb{V}\left(\mathbb{1}_{A_{n}}\right)
$$

Estimating the first sum gives

$$
\begin{aligned}
\sum_{n: d_{n}=1} \mathbb{V}\left(\mathbb{1}_{A_{n}}\right) & \geq \mathfrak{m}\left(a_{n}=1\right) \cdot \sum_{n: d_{n}=1} \mathfrak{m}\left(A_{n}^{c}\right) \geq \frac{\mathfrak{m}\left(a_{n}=1\right)}{\log 2} \cdot \sum_{n: d_{n}=1} \lambda\left(a_{n}>1+1 / c_{n}\right) \\
& >\frac{\mathfrak{m}\left(a_{n}=1\right)}{\log 2} \cdot \sum_{n: d_{n}=1} \frac{c_{n}}{2+c_{n}}
\end{aligned}
$$

and $\sum_{n: d_{n}=1} c_{n}=\infty$ implies (35). Estimating the second sum gives

$$
\sum_{n: d_{n}>1} \mathbb{V}\left(\mathbb{1}_{A_{n}}\right) \geq \sum_{n: d_{n}>1} \mathfrak{m}\left(a_{n}=1\right) \cdot \mathfrak{m}\left(A_{n}\right) \geq \frac{\mathfrak{m}\left(a_{1}=1\right)}{\log 2} \sum_{n: d_{n}>1} \lambda\left(A_{n}\right)
$$

and by (20) and (21) each of $\sum_{n: d_{n}>1} 1 / d_{n}^{2}$ and $\sum_{n: d_{n}>1} 1 /\left(c_{n} d_{n}\right)$ implies (35).

ad (C): We have that

$$
\sum_{n=1}^{\infty} \mathbb{V}\left(\mathbb{1}_{A_{n}}\right) \geq \sum_{n: c_{n}>d_{n}} \mathfrak{m}\left(a_{n}=1\right) \cdot \mathfrak{m}\left(A_{n}\right) \geq \frac{\mathfrak{m}\left(a_{1}=1\right)}{\log 2} \cdot \sum_{n: c_{n}>d_{n}} \lambda\left(A_{n}\right)
$$

and by (22) the condition $\sum_{n: c_{n}>d_{n}} 1 /\left(c_{n} d_{n}\right)=\infty$ implies (35).

\section{ACKNowledgements}

We thank Sam Chow for pointing out to us that the value of the first $\phi$-mixing coefficient coincides with the Erdős-Ford-Tenenbaum constant. 


\section{REFERENCES}

[Ber11] F. Bernstein. Über eine Anwendung der Mengenlehre auf ein aus der Theorie der säkularen Störungen herrührendes Problem. Math. Ann., 71(3):417-439, 1911.

[Ber12] F. Bernstein. Über geometrische Wahrscheinlichkeit und über das Axiom der beschränkten Arithmetisierbarkeit der Beobachtungen. Math. Ann., 72(4):585-587, 1912.

[Bor09] E. Borel. Les probabilités denombrables et leurs applications arithmétiques. Rend. Circ. Mat. Palermo, 27:247271, 1909.

[Bra05] R. C. Bradley. Basic properties of strong mixing conditions. A survey and some open questions. Probability Surveys, 2:107-144, 2005.

[BvdPSZ14] J. Borwein, A. van der Poorten, J. Shallit, and W. Zudilin. Neverending fractions, volume 23 of Australian Mathematical Society Lecture Series. Cambridge University Press, Cambridge, 2014. An introduction to continued fractions.

[DK02] K. Dajani and C. Kraaikamp. Ergodic Theory of Numbers. The Mathematical Association of America, July 2002.

[FLWW09] A.-H. Fan, L.-M. Liao, B.-W. Wang, and J. Wu. On Khintchine exponents and Lyapunov exponents of continued fractions. Ergodic Theory Dynam. Systems, 29(1):73-109, 2009.

[For08] K. Ford. The distribution of integers with a divisor in a given interval. Ann. of Math. (2), 168(2):367-433, 2008.

[Gal72] J. Galambos. Some remarks on the Lüroth expansion. Czechoslovak Mathematical Journal, 22(2):266-271, 1972 .

[IK09] M. Iosifescu and C. Kraaikamp. Metrical Theory of Continued Fractions. Springer Netherlands, 2002. edition, December 2009.

[JP18] O. Jenkinson and M. Pollicott. Rigorous effective bounds on the Hausdorff dimension of continued fraction Cantor sets: A hundred decimal digits for the dimension of $E_{2}$. Adv. Math., 325:87-115, 2018.

[Khi35] A. Y. Khintchine. Metrische Kettenbruchprobleme. Compositio Math, 1:361-382, 1935.

[Khi64] A. Ya. Khintchine. Continued fractions. The University of Chicago Press, Chicago, Ill.-London, 1964.

[KMS16] M. Kesseböhmer, S. Munday, and B. O. Stratmann. Infinite Ergodic Theory of Numbers. De Gruyter Graduate. De Gruyter, Berlin, 2016.

[KS07] Kesseböhmer, M., Stratmann, B.O.: A multifractal analysis for Stern-Brocot intervals, continued fractions and Diophantine growth rates. J. Reine Angew. Math. 605:133-163, 2007.

[KZ06] Kesseböhmer, M., Zhu, S.: Dimension sets for infinite IFSs: the Texan conjecture. J. Number Theory 116(1):230-246, 2006.

[LR16] L. Liao and M. Rams. Upper and lower fast Khintchine spectra in continued fractions. Monatsh. Math., 180(1):65-81, 2016.

[Phi67] W. Philipp. Some metrical theorems in number theory. Pacific Journal of Mathematics, 20(1):109-127, 1967.

[Phi70] W. Philipp. Some metrical theorems in number theory. II. Duke Math. J., 37:447-458, 1970.

[Phi88] W. Philipp. Limit theorems for sums of partial quotients of continued fractions. Monatshefte für Mathematik, 105(3):195-206, 1988.

[PW73] W. Philipp and G. R. Webb. An invariance principle for mixing sequences of random variables. Z. Wahrscheinlichkeitstheorie und Verw. Gebiete, 25:223-237, 1973.

[Sam85] J. D. Samur. A note on the convergence to Gaussian laws of sums of stationary $\phi$-mixing triangular arrays. In Probability in Banach spaces, V (Medford, Mass., 1984), volume 1153 of Lecture Notes in Math., pages 387-399. Springer, Berlin, 1985.

[WW08] B.-W. Wang and J. Wu. Hausdorff dimension of certain sets arising in continued fraction expansions. Adv. Math., 218(5):1319-1339, 2008.

[Zup81] T. M. Zuparov. On a theorem from the metric theory of continued fractions. Izv. Akad. Nauk UzSSR Ser. Fiz.-Mat. Nauk, (6):9-12, 78, 1981.

Universität Bremen, Fachbereich 3 - Mathematik und Informatik, Bibliothekstr. 5, 28359 Bremen, Germany

E-mail address: mhk@math.uni-bremen.de

Australian National University, Research School of Finance, Actuarial Studies and Statistics, 26C Kingsley St, ACton ACT 2600, Australia

E-mail address: tanja.schindler@anu.edu.au 